\title{
SIGNIFICANCE OF YEASTS IN BLOODSTREAM INFECTION: EPIDEMIOLOGY AND PREDISPOSING FACTORS OF CANDIDAEMIA IN ADULT PATIENTS AT A UNIVERSITY HOSPITAL (2010-2014)
}

\author{
JÚliA PONGRÁCZ*, EMESE JUHÁSZ, \\ MiKLÓs IVÁN and KATALIN KRISTÓF \\ Clinical Microbiology Laboratory, Institute of Laboratory Medicine, \\ Semmelweis University, Budapest, Hungary
}

(Received: 23 June 2015; accepted: 6 July 2015)

\begin{abstract}
The incidence of Candida bloodstream infection (BSI) has increased during the past decades. Species distribution is changing worldwide, and non-albicans Candida spp. are becoming more prevalent. Acquired resistance to antifungal agents has been documented in several reports. The aim of our study was to assess the epidemiology and antifungal susceptibility of Candida isolates from BSI at our institute. The incidence of Candida BSI increased during the first four years of our investigation, from 1.7 to 3.5 episodes / 10000 admissions, then dropped to 2.66 episodes / 10000 admissions in the last year. The most frequently isolated species was C. albicans $(63 \%)$, followed by C. glabrata (13\%), C. parapsilosis $(10.2 \%)$, C. tropicalis (9.3\%), and C. krusei (3.7\%). One isolate each of C. kefyr, C. fabianii and C. inconspicua were detected. The percentage of $C$. albicans remained stable throughout the study period. The most frequent risk factors of Candida BSI in our patient population were intensive care treatment $(60.4 \%)$, abdominal surgery $(52.5 \%)$, and solid malignancy $(30.7 \%)$. All isolates were wild-type organisms, no acquired antifungal resistance was detected.
\end{abstract}

Keywords: Candida spp., bloodstream infection, epidemiology, antifungal susceptibility

\section{Introduction}

Candida spp. are a leading cause of bloodstream infection (BSI), associated with high mortality and increased hospital costs [1]. The annual incidence of Candida BSI varies greatly by region based on epidemiological studies from

*Corresponding author; E-mail: pongraczjulia@yahoo.com 
Europe and the USA: incidence ranges from 3.0 to 26.2 / 100000 inhabitants. The incidence of Candida BSI in Denmark and Spain were similar (8.6 / 100000 inhabitants, and 8.1 / 100000 inhabitants, respectively), while a much higher incidence (26.2 / 100000 inhabitants) was reported from Baltimore in the USA, and the incidence was the lowest in Norway (3.0 / 100000 inhabitants) [2-6].

Risk factors of Candida BSI are well established, including treatment at the intensive care unit (ICU), broad-spectrum antibiotic therapy, abdominal surgery, malignancy, the administration of total parenteral nutrition (TPN), the presence of intravenous catheters, mechanical ventilation, acute renal failure, immunosuppressive treatment, neutropenia, and prior colonization with Candida spp. [7-12].

C. albicans remains the most frequently isolated species in most reports, but a shift towards non-albicans species has been detected $[6,13,14]$. The rise in the rate of fluconazole non-susceptible Candida spp., mainly C. glabrata, has been linked with prior fluconazole exposure $[15,16]$. Reports of breakthrough Candida BSI and the isolation of multiresistant isolates, especially C. glabrata strains resistant to echinocandins, are alarming [17, 18].

The epidemiology of Candida BSIs varies greatly by region, therefore evaluation of local data is essential to assess local trends, identify patients at risk of infection, and to determine which antifungal drug is most adequate as empirical therapy. The aim of our study was to evaluate the epidemiology of Candida BSIs in the adult population at our institute, and to detect any strains with acquired antifungal resistance.

\section{Materials and Methods}

All episodes of candidaemia in patients over the age of 18 were analyzed during a five-year period, from January 2010 to December 2014, at Semmelweis University, a 2250-bed tertiary hospital with 105000 admissions per year. An episode of candidaemia was defined as isolation of Candida from blood culture on at least one occasion. Episodes were considered separate if they occurred more than 30 days apart, or different Candida species were isolated.

Patient data was collected retrospectively using a standardized case report form which included demographic data, underlying diseases, comorbidities (diabetes mellitus, organ failure, malignancy, immunosuppression), presence of intravascular devices, mechanical ventilation, administration of parenteral nutrition, and isolate characteristics (Candida species, antifungal susceptibility profile). Microbiological data, such as previous colonization by Candida spp., and time to blood culture positivity were also recorded. 
Blood samples were collected and processed using either the Bactec ${ }^{\mathrm{TM}} 9120$ (Beckton Dickinson) or BacT/ALERT ${ }^{\circledR} 3 \mathrm{D}$ (BioMérieux) automated blood culture systems. Isolates were identified by phenotypic methods [carbohydrate assimilation profile obtained by the API 20C AUX system (BioMérieux), and morphology of pseudohyphae on malt agar] and matrix-assisted laser desorption/ ionization time-of-flight mass spectrometry (MALDI-TOF/MS, Bruker) analysis. Routine antifungal susceptibility testing was performed by E-test ${ }^{\circledR}$ (BioMérieux) method, according to the manufacturer's instructions. Minimal inhibitory concentration (MIC) results were interpreted according to EUCAST clinical breakpoints [19].

Fisher's exact test was used to assess if any of the risk factors of candidaemia had any connection with 30-day mortality and to describe changes in isolate incidence. A p value of $<0.05$ was considered significant.

\section{Results}

\section{Incidence}

Candidaemia was detected in 129 cases in adult patients between 20102014. The number of episodes per year and their incidence is presented in Table I. More than half $(62 \%, n=80)$ of the 129 episodes occurred in the ICU. Figure 1 shows the distribution of candidaemia in the different departments.

\section{Patient demographics and clinical characteristics}

The mean age of patients was 62.8 years overall, and $60.1 \%$ were male. Eighty-three (64.8\%) patients were admitted with a surgical diagnosis, and $55.5 \%$ of the patients had major abdominal surgery before the detection of candidaemia.

Table I. Candidaemia in adult patients at Semmelweis University, 2010-2014

\begin{tabular}{lcc}
\hline & Number of episodes & 10000 admissions \\
\hline 2010 & 18 & 1.7 \\
2011 & 19 & 1.8 \\
2012 & 27 & 2.57 \\
2013 & 37 & 3.50 \\
2014 & 28 & 2.66 \\
\hline
\end{tabular}




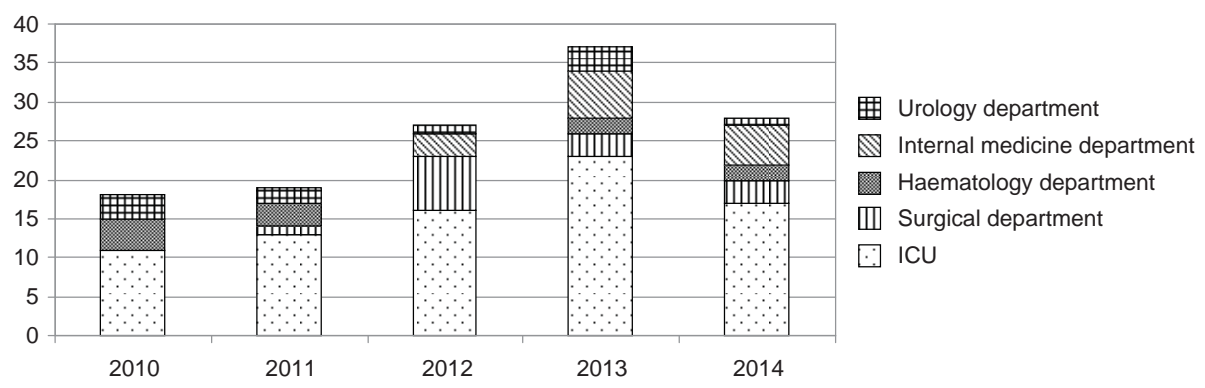

Figure 1. Incidence of candidaemia (number of episodes per department) at Semmelweis University, 2010-2014

Among underlying diseases of Candida BSI, solid malignancy was the most prevalent $(30.5 \%)$, followed by diabetes mellitus $(24.2 \%)$, and immunosuppression $(19.5 \%)$.

The mean time to blood culture positivity was 1.41 days overall (range 0.37-5.64 days), with blood cultures containing C. glabrata requiring longer incubation time to positivity (2.67 days on average, range $0.43-5.64)$ than the other species.

Previous colonization in at least one site by the same Candida sp. that was isolated from the bloodstream was detected in 99 episodes $(77.3 \%)$. The sites of colonization were the respiratory tract $(46.9 \%)$, the urinary tract $(22.6 \%)$, an intravascular device (35.9\%), and wounds (23.4\%). The source of Candida BSI was identified in 74 cases: four bloodstream isolates originated from the urinary tract, 17 from an abdominal abscess or peritoneal infection, three from deep skin infections, three from the mediastinum, and one case of endocarditis was identified. Forty-six (35.9\%) episodes were associated with a central venous line. Demographic data and clinical characteristics are summarized in Table II.

Overall 30-day mortality was $50.8 \%$, whereas 30 -day mortality the highest (90.9\%) with C. tropicalis BSI and the lowest with C. krusei $(25 \%)$ and C. parapsilosis $(27.3 \%)$.

Univariate analysis was performed to assess if any risk factors of candidaemia could be associated with a higher mortality rate. No such connection was detected; $p$ values are presented in Table III.

\section{Isolate characteristics}

A total of 136 isolates were collected. The most frequently isolated species was C. albicans (63\%), followed by C. glabrata (13\%), C. parapsilosis (10.2\%), C. tropicalis (9.3\%), and C. krusei (3.7\%). C. kefyr caused one episode 
in 2013, and C. fabianii and C. inconspicua caused one episode each in 2014. Seven cases (6.9\%) of BSI with multiple Candida spp. were recorded. C. albicans and $C$. glabrata were isolated in four cases, C. albicans and C. parapsilosis in one case, $C$. albicans and $C$. tropicalis in one case, and $C$. parapsilosis and C. glabrata in one case.

There was no significant change in the percentage of $C$. albicans per year (63\% of all isolates) during the study period, but the rate of species with decreased susceptibility or resistance to fluconazole increased from $10.5 \%$ in 2010 to $39.3 \%$ in $2014(\mathrm{p}<0.005)$. Figure 2 shows species distribution between 2010 and 2014.

All isolates were wild-type organisms and no acquired resistance was detected based on EUCAST breakpoints and available epidemiological cut-off values. The antifungal MIC range, MIC 50 and MIC 90 values are presented in Table IV.

\section{Discussion}

The incidence of Candida BSI at our institute increased between 2010 and $2013\left(\mathrm{R}^{2}=0.9\right)$ from 1.7 to 3.5 episodes / 10000 admissions, in agreement with worldwide trends, but dropped back to 2.66 episodes / 10000 admissions in 2014 . The incidence rate is similar to previous Hungarian data and results from other European centers $[20,21,22]$. The decrease in incidence during the last study year may be explained by the administration of prophylactic antifungal therapy by clinicians after the problem of increasing rates of Candida BSI was detected.

C. albicans was the most frequently isolated species (63\%), and the percentage of $C$. albicans isolates per year did not change significantly during the study period. Several studies have detected a shift towards non-albicans Candida spp., especially C. glabrata, causing BSI [23]. This trend was observed at our institute as well, with the number of non-albicans isolates increasing steadily from 2010 to 2014. The number of C. glabrata isolates increased from 2 isolates in 2010 to 9 isolates in 2014.

Candidaemia was especially prevalent in patients in the ICU after abdominal surgery: the majority of our cases $(60.9 \%)$ occurred in the ICU, and over half $(55.5 \%)$ following major abdominal surgery. These findings mirror the results of Kuhns et al., who in a survey of 47707 cases determined that $20.7 \%$ of BSI in the ICU following abdominal surgery were caused by Candida spp. [24].

Solid malignancy was a major risk factor of candidaemia, present in $30.5 \%$ of the patients. Surveillance cultures are recommended in patients at risk to detect colonization by Candida spp. [25], which precluded Candida BSI at our institute in $77.3 \%$ of cases. 


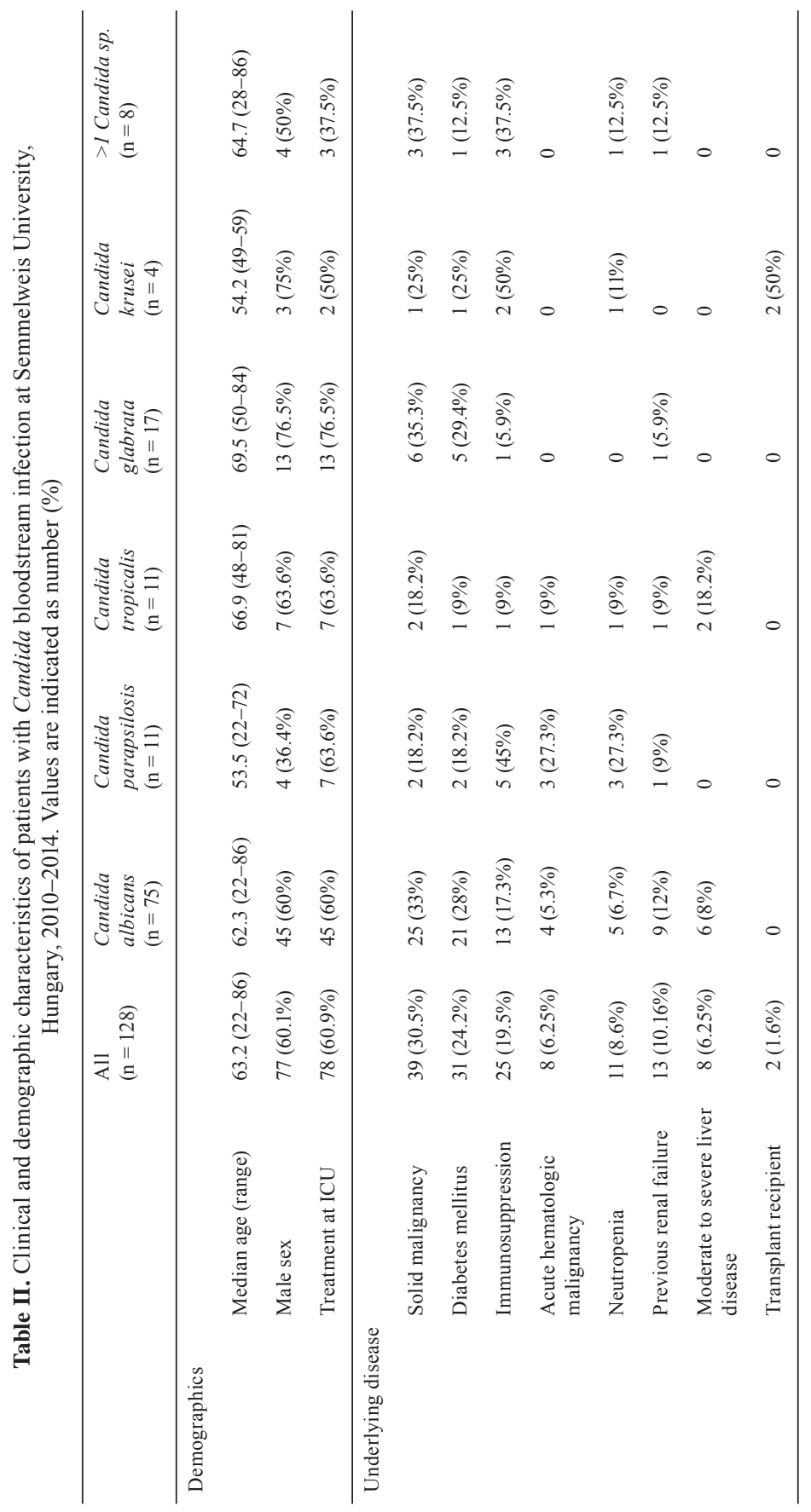




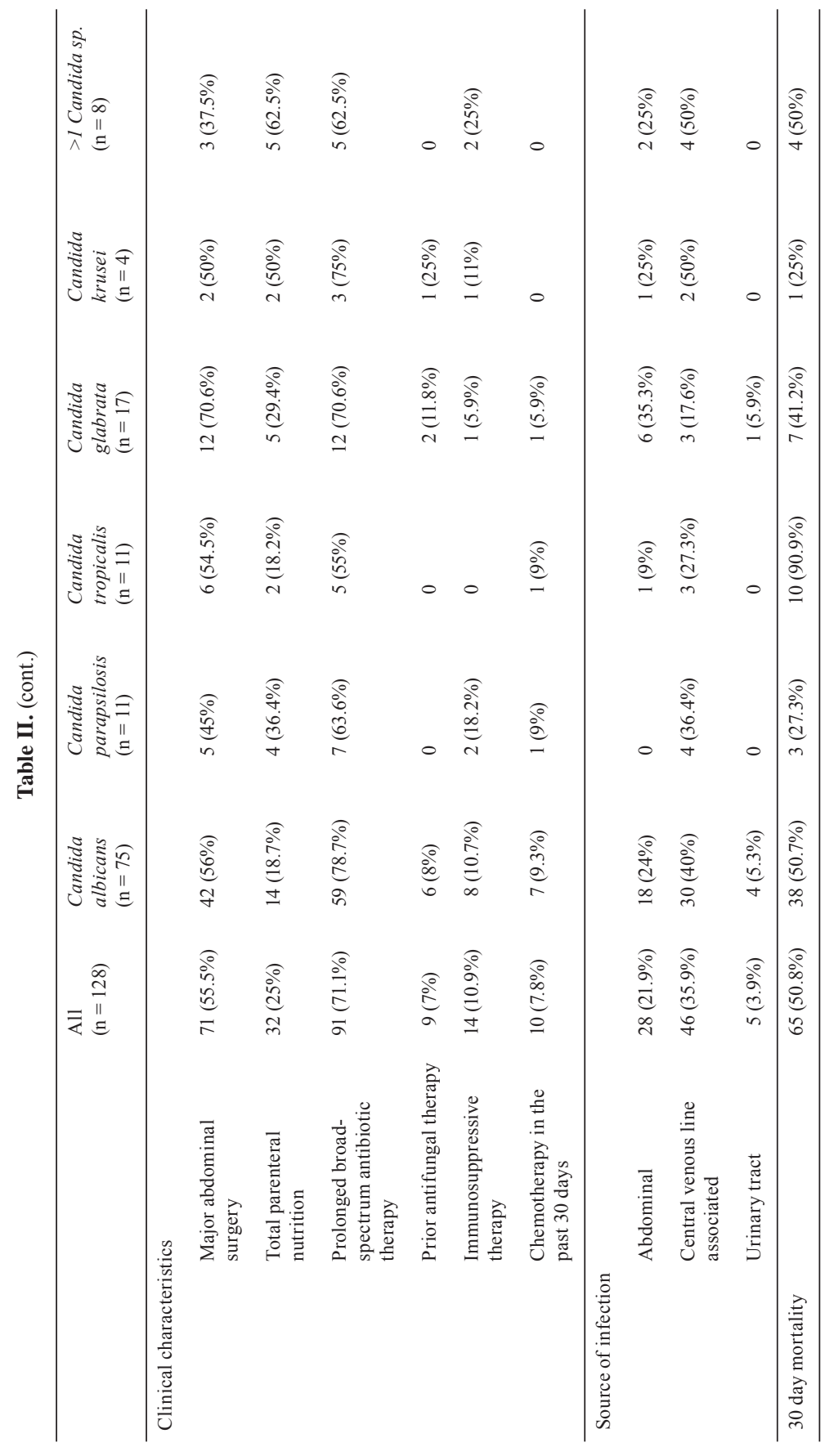


Table III. Risk factors of candidaemia vs. mortality (2010-2014, Semmelweis University, Hungary)

\begin{tabular}{lccc}
\hline & Recovered patient & Fatal outcome & p value \\
\hline Abdominal surgery & 27 & 24 & 0.2 \\
Treatment at ICU & 31 & 27 & 0.088 \\
Solid malignancy & 11 & 15 & 0.652 \\
Acute leukaemia & 4 & 3 & 0.7 \\
Immunosuppression & 8 & 12 & 0.617 \\
Parenteral nutrition & 11 & 11 & 0.808 \\
Presence of an endovascular catheter & 27 & 36 & 0.656 \\
\hline
\end{tabular}

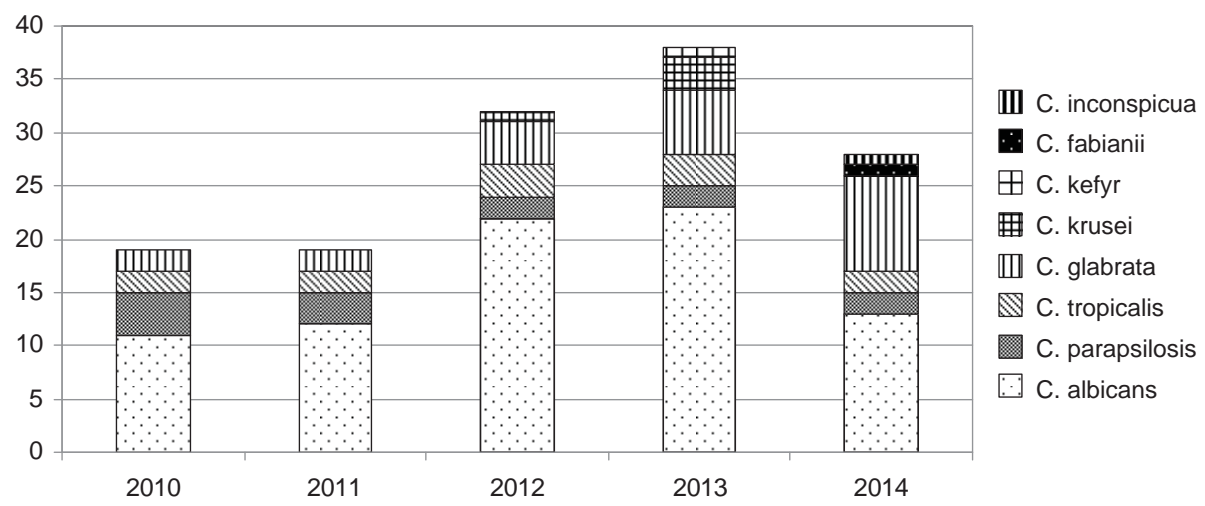

Figure 2. Species distribution of Candida bloodstream isolates at Semmelweis University, Hungary, 2010-2014

Table IV. Antifungal MIC range, MIC50 and MIC90 values of Candida bloodstream isolates at Semmelweis University, Hungary, 2010-2014

\begin{tabular}{lllll}
\hline Species & Antifungal agent & MIC range $(\mu \mathrm{g} / \mathrm{ml})$ & MIC50 $(\mu \mathrm{g} / \mathrm{ml})$ & MIC90 $(\mu \mathrm{g} / \mathrm{ml})$ \\
\hline $\begin{array}{l}\text { Candida albicans } \\
(\mathrm{n}=75)\end{array}$ & Fluconazole & $0.064-1$ & 0.25 & 0.5 \\
& Itraconazole & $0.016-0.125$ & 0.032 & 0.125 \\
& Voriconazole & $0.004-0.064$ & 0.008 & 0.064 \\
& Posaconazole & $0.008-0.016$ & 0.008 & 0.016 \\
& Anidulafungin & $0.008-0.032$ & 0.016 & 0.032 \\
& Micafungin & $0.004-0.016$ & 0.008 & 0.016 \\
& Caspofungin & $0.004-0.032$ & 0.008 & 0.032 \\
& Amphotericin B & $0.064-0.5$ & 0.25 & 0.5 \\
\hline
\end{tabular}


Table IV. (cont.)

\begin{tabular}{|c|c|c|c|c|}
\hline Species & Antifungal agent & $\mathrm{MIC}$ range $(\mu \mathrm{g} / \mathrm{ml})$ & $\operatorname{MIC50}(\mu \mathrm{g} / \mathrm{ml})$ & $\operatorname{MIC} 90(\mu \mathrm{g} / \mathrm{ml})$ \\
\hline \multirow{8}{*}{$\begin{array}{l}\text { Candida parapsilosis } \\
(\mathrm{n}=11)\end{array}$} & Fluconazole & $0.064-1$ & 0.125 & 0.5 \\
\hline & Itraconazole & $0.03-0.125$ & 0.125 & 0.125 \\
\hline & Voriconazole & $0.004-0.064$ & 0.016 & 0.032 \\
\hline & Posaconazole & $0.008-0.032$ & 0.016 & 0.032 \\
\hline & Anidulafungin & $0.5-1.0$ & 1 & 1 \\
\hline & Micafungin & $0.25-0.5$ & 0.25 & 0.5 \\
\hline & Caspofungin & $0.064-0.25$ & 0.125 & 0.25 \\
\hline & Amphotericin B & $0.125-0.5$ & 0.25 & 0.5 \\
\hline \multirow{8}{*}{$\begin{array}{l}\text { Candida tropicalis } \\
(\mathrm{n}=11)\end{array}$} & Fluconazole & $0.125-1$ & 0.25 & 0.5 \\
\hline & Itraconazole & $0.016-0.125$ & 0.064 & 0.125 \\
\hline & Voriconazole & $0.008-0.064$ & 0.016 & 0.064 \\
\hline & Posaconazole & $0.008-0.032$ & 0.016 & 0.032 \\
\hline & Anidulafungin & $0.008-0.016$ & 0.016 & 0.016 \\
\hline & Micafungin & $0.008-0.032$ & 0.016 & 0.032 \\
\hline & Caspofungin & $0.016-0.032$ & 0.032 & 0.032 \\
\hline & Amphotericin B & $0.125-0.5$ & 0.25 & 0.5 \\
\hline \multirow{8}{*}{$\begin{array}{l}\text { Candida glabrata } \\
(\mathrm{n}=17)\end{array}$} & Fluconazole & $2.0-16$ & 4 & 8 \\
\hline & Itraconazole & $0.064-0.25$ & 0.125 & 0.25 \\
\hline & Voriconazole & $0.125-0.5$ & 0.25 & 0.5 \\
\hline & Posaconazole & $0.5-1.0$ & 1 & 1 \\
\hline & Anidulafungin & $0.016-0.03$ & 0.03 & 0.03 \\
\hline & Micafungin & $0.004-0.016$ & 0.08 & 0.016 \\
\hline & Caspofungin & $0.004-0.03$ & 0.016 & 0.03 \\
\hline & Amphotericin B & $0.25-1.0$ & 0.5 & 1 \\
\hline \multirow{8}{*}{$\begin{array}{l}\text { Candida krusei } \\
(\mathrm{n}=4)\end{array}$} & Fluconazole & $16-32$ & 32 & 32 \\
\hline & Itraconazole & $0.03-1.0$ & 1 & 1 \\
\hline & Voriconazole & $0.032-1.0$ & 0.064 & 1 \\
\hline & Posaconazole & $0.032-0.125$ & 0.032 & 0.125 \\
\hline & Anidulafungin & $0.008-0.03$ & 0.016 & 0.03 \\
\hline & Micafungin & $0.004-0.03$ & 0.008 & 0.03 \\
\hline & Caspofungin & $0.016-0.03$ & 0.016 & 0.03 \\
\hline & Amphotericin B & $0.25-0.5$ & 0.25 & 0.5 \\
\hline
\end{tabular}


Eight cases $(6.25 \%)$ of BSI caused by more than one Candida spp. were detected. Polyfungal candidaemia is uncommon and has been associated with severe underlying disease, use of intravascular catheter, treatment at ICU, prior antibiotic exposure and heavy colonization with Candida spp. [26]. Significant statistical results could not be achieved due to the small sample size, but patients with multiple Candida isolates suffered from solid malignant disease $(37.5 \%)$, and received TPN more frequently (62.5\%) compared with overall rates.

Acquired resistance to at least one group of antifungals has been detected in case of several Candida spp. Reports of multi-resistant C. glabrata isolates resistant to azoles, echinocandins and amphotericin B are especially alarming $[27,28]$. All the isolates in our study were wild-type organisms, indicating that acquired antifungal resistance of Candida spp. is not an issue in Hungary yet. The emergence of resistant isolates has been linked with prior antifungal treatment. Echinocandin treatment has only recently been implemented in Hungarian routine clinical care, explaining why resistant isolates have not been detected yet. What is especially daunting is the fact that isolates resistant to echinocandins are frequently resistant to amphotericin B as well, posing a serious therapeutic challenge to clinicians.

The overall 30 -day mortality rate at our institute was higher $(50.8 \%)$ than figures reported in other European and US centers (22-37\%) [29, 30]. Mortality rate by species was the highest for $C$. tropicalis $(90.9 \%)$ and $C$. albicans $(50.7 \%)$; these two species are the most virulent compared with other species and have been associated with higher mortality rates in BSI [31], though the mortality rate for $C$. tropicalis may be falsely high due to the small sample size.

No risk factors of Candida BSI were found to be significantly associated with patient mortality.

This study describes the local epidemiology and antifungal susceptibility at a university hospital. The incidence of Candida BSI increased during the study period, and fluconazole non-susceptible species became more prevalent. Rare Candida spp., such as C. fabianii and C. inconspicua were identified as cause of BSI. No isolates with acquired antifungal resistance were detected. Surveillance of Candida BSI should be continued in the future to assess changes in epidemiology and to monitor antifungal resistance.

\section{Authors' Contributions}

PJ and KK conceived and designed the study. PJ collected and analysed the data. PJ and KK wrote and corrected the manuscript. JE and IM contributed in collecting the isolates and in correction of the manuscript. 


\section{Conflict of Interest}

The authors declare that they have no competing interests.

\section{References}

1. Kett, D. H., Azoulay, E., Echeverria, P. M., Vincent, J. L., and for the Extended Prevalence of Infection in the ICU Study (EPIC II) Group of Investigators: Candida bloodstream infections in intensive care units: Analysis of the extended prevalence of infection in intensive care unit study. Crit Care Med 39, 665-670 (2011).

2. Arendrup, M. C., Bruun, B., Christensen, J. J., Fuursted, K., Johansen, H. K., Kjaeldgaard, P., Knudsen, J. D., Kristensen, L., Møller, J., Nielsen, L., Rosenvinge, F. S., Røder, B., Schønheyder, H. C., Thomsen, M. K., Truberg, K.: National surveillance of fungemia in Denmark (2004 to 2009). J Clin Microbiol 49, 325-334 (2011).

3. Puig-Asensio, M., Padilla, B., Garnacho-Montero, J., Zaragoza, O., Aguado, J. M., Zaragoza, R., Montejo, M., Muñoz, P., Ruiz-Camps, I., Cuenca-Estrella, M., Almirante, B.; CANDIPOP Project; GEIH-GEMICOMED (SEIMC); REIPI: Epidemiology and predictive factors for early and late mortality in Candida bloodstream infections: A population-based surveillance in Spain. Clin Microbiol Infect 20, 245-254 (2014).

4. Sandven, P., Bevanger, L., Digranes, A., Haukland, H. H., Mannsåker, T., Gaustad, P., and the Norwegian Yeast Study Group: Candidemia in Norway (1991 to 2003): Results from a nationwide study. J Clin Microbiol 44, 1977-1981 (2006).

5. Cleveland, A. A., Farley, M. M., Harrison, L. H., Stein, B., Hollick, R., Lockhart, S. R., Magill, S. S., Derado, G., Park, B. J., Chiller, T. M.: Changes in incidence and antifungal drug resistance in candidemia: Results from population-based laboratory surveillance in Atlanta and Baltimore, 2008-2011. Clin Infect Dis 55, 1352-1361 (2012).

6. Wisplinghoff, H., Ebbersa, J., Geurtza, L., Stefanik, D., Major, Y., Edmond, M. B., Wenzel, P., Seifert, H.: Nosocomial bloodstream infections due to Candida spp. in the USA: Species distribution, clinical features and antifungal susceptibilities. Int J Antimicrob Agents 43, 78-81 (2014).

7. Tapia, G. G., Razonable, R. R., Eckel-Passow, J. E., Lahr, B. D., Afessa, B., Keegan, M. T., Catania, J., Baddour, L. M.: A scoring model of factors associated with Candida glabrata candidemia among critically ill patients. Mycoses 55, 228-236 (2012).

8. Chi, H. W., Yang, Y. S., Shang, S. T., Chen, K. H., Yeh, K. M., Chang, F. Y., Lin, J. C.: Candida albicans versus non-albicans bloodstream infections: The comparison of risk factors and outcome. J Microbiol Immunol Infect 44, 369-375 (2011).

9. Leenders, N. H., Oosterheert, J. J., Ekkelenkamp, M. B., De Lange, D. W., Hoepelman, A. I., Peters, E. J.: Candidemic complications in patients with intravascular catheters colonized with Candida species: An indication for preemptive antifungal therapy? Int J Infect Dis 15, 453-458 (2011).

10. Pfaller, M., Neofytos, D., Diekema, D., Azie, N., Meier-Kriesche, H. U., Quan, S. P., Horn, D.: Epidemiology and outcomes of candidemia in 3648 patients: Data from the Prospective Antifungal Therapy (PATH Alliance ${ }^{\circledR}$ ) registry, 2004-2008. Diagn Microbiol Infect Dis 74, 323-331 (2012). 
11. Jordá-Marcos, R., Álvarez-Lerma, F., Jurado, M., Palomar, M., Nolla-Salas, J., León, M. A., León, C.; EPCAN Study Group: Risk factors for candidaemia in critically ill patients: A prospective surveillance study. Mycoses 50, 302-310 (2007).

12. Labelle, A. J., Micek, S. T., Roubinian, N., Kollef, M. H.: Treatment-related risk factors for hospital mortality in Candida bloodstream infections. Crit Care Med 36, $2967-$ 2972 (2008).

13. Hii, I. M., Chang, H. L., Lin, L. C., Lee, Y. L., Liu, Y. M., Liu, C. E., Chen, C. H., Cheng, Y. R., Chang, C. Y.: Changing epidemiology of candidemia in a medical center in middle Taiwan. J Microbiol Immunol Infect, http://dx.doi.org/10.1016/j.jmii.2013.08.017 (2013).

14. Ma, C. F., Li, F. Q., Shi, L. N., Hu, Y. A., Wang, Y., Huang, M., Kong, Q. Q.: Surveillance study of species distribution, antifungal susceptibility and mortality of nosocomial candidemia in a tertiary care hospital in China. BMC Infect Dis 13, 337 (2013).

15. Bassetti, M., Ansaldi, F., Nicolini, L., Malfatto, E., Molinari, M. P., Mussap, M., Rebesco, B., Bobbio Pallavicini, F., Icardi, G., Viscoli, C.: Incidence of candidaemia and relationship with fluconazole use in an intensive care unit. J Antimicrob Chemother 64, 625629 (2009).

16. Lee, I., Fishman, N. O., Zaoutis, T. E., Morales, K. H., Weiner, M. G., Synnestvedt, M., Nachamkin, I., Lautenbach, E.: Risk factors for fluconazole-resistant Candida glabrata bloodstream infections. Arch Intern Med 169, 379-383 (2009).

17. Pfeiffer, C. D., Garcia-Effron, G., Zaas, A. K., Perfect, J. R., Perlin, D. S., Alexander, B. D.: Breakthrough invasive Candidiasis in patients on micafungin. J Clin Microbiol 48, 2373-2380 (2010).

18. Cho, E. J., Shin, J. H., Kim, S. H., Kim, H. K., Park, J. S., Sung, H., Kim, M. N., Im, H. J.: Emergence of multiple resistance profiles involving azoles, echinocandins and amphotericin B in Candida glabrata isolates from a neutropenia patient with prolonged fungaemia. J Antimicrob Chemother 70, 1268-1270 (2015).

19. The European Committee on Antimicrobial Susceptibility Testing. Antifungal Agents. Breakpoint tables for interpretation of MICs. Version 7.0, 2014. http://www.eucast.org.

20. Wille, M. P., Guimarães, T., Furtado, G. H., Colombo, A. L.: Historical trends in the epidemiology of candidaemia: Analysis of an 11-year period in a tertiary care hospital in Brazil. Mem Inst Oswaldo Cruz 108(3), 288-292 (2013).

21. Dóczi, I., Dósa, E., Hajdú, E., Nagy, E.: Aetology and antifungal susceptibility of yeast bloodstream infections in a Hungarian university hospital between 1996 and 2000. I Med Microbiol 51(8), 677-681 (2002).

22. Tortorano, A. M., Peman, J., Bernhardt, H., Klingspor, L., Kibbler, C. C., Faure, O., Biraghi, E., Canton, E., Zimmermann, K., Seaton, S., Grillot, R.; ECMM Working Group on Candidaemia: Epidemiology of candidaemiain Europe: Results of 28-month European Confederation of Medical Mycology (ECMM) hospital-based surveillance study. Eur J Clin Microbiol Infect Dis 23(4), 317-322 (2004).

23. Moretti, M. L., Trabasso, P., Lyra, L., Fagnani, R., Resende, M. R., de Oliveira Cardoso, L. G., Schreiber, A. Z.: Is the incidence of candidemia caused by Candida glabrata increasing in Brazil? Five-year surveillance of Candida bloodstream infection in a university reference hospital in southeast Brazil. Med Mycol 51(3), 225-230 (2013).

24. Kuhns, M., Rosenberger, A., Bader, O., Reichard, U., Gross, U., Weig, M.: Incidence of Candidaemia following abdominal surgery in German hospitals. Zentralbl Chir 2013 Nov 15. [in German] 
25. Posteraro, B., De Pascale, G., Tumbarello, M., Torelli, R., Pennisi, M. A., Bello, G., Maviglia, R., Fadda, G., Sanguinetti, M., Antonelli, M.: Early diagnosis of candidemia in intensive care unit patients with sepsis: a prospective comparison of (1-3)- $\beta$-D-glucan assay, Candida score, and colonization index. Crit Care 15(5), R249 (2011).

26. Pulimood, S., Ganesan, L., Alangaden, G., Chandrasekar, P.: Polymicrobial candidemia. Diagn Microbiol Infect Dis 44(4), 353-357 (2002)

27. Cho, E. J., Shin, J. H., Kim, S. H., Kim, H. K., Park, J. S., Sung, H., Kim, M. N., Im, H. J.: Emergence of multiple resistance profiles involving azoles, echinocandins and amphotericin B in Candida glabrata isolates from a neutropenia patient with prolonged fungaemia. J Antimicrob Chemother 70(4), 1268-1270 (2015).

28. Cleveland, A. A., Harrison, L. H., Farley, M. M., Hollick, R., Stein, B., Chiller, T. M., Lockhart, S. R., Park, B. J.: Declining incidence of Candidemia and the shifting epidemiology of candida resistance in two US metropolitan areas, 2008-2013: Results from population-based surveillance. PLoS One 10(3), e0120452 (2015).

29. Pfaller, M., Neofytos, D., Diekema, D., Azie, N., Meier-Kriesche, H. U., Quan, S. P., Horn, D.: Epidemiology and outcomes of candidemia in 3648 patients: Data from the Prospective Antifungal Therapy (PATH Alliance ${ }^{\circledR}$ ) registry, 2004-2008. Diagn Microbiol Infect Dis 74, 323-331 (2012).

30. Das, I., Nightingale, P., Patel, M., Jumaa, P.: Epidemiology, clinical characteristics, and outcome of candidemia: Experiencein a tertiary referral center in the UK. Int J Infect Dis 15(11), 759-763 (2011).

31. Arendrup, M., Horn, T., Frimodt-Møller, N.: In vivo pathogenicity of eight medically relevant Candida species in an animal model. Infection 30(5), 286-291 (2002). 